\title{
Retrato do perfil de saúde-doença de idosos longevos usuários da atenção básica de saúde
}

\author{
Profiling health and disease in oldest-old primary health care users \\ Retrato del perfil de salud-enfermedad de ancianos longevos usuarios de atención primaria de salud
}

\author{
Letice de Freitas Pereira'; Maria Helena Lenardt ${ }^{I I}$; Tatiane Michel ${ }^{I I I}$; \\ Nathalia Hammerschmidt Kolb Carneiro ${ }^{I V}$; Leandra de Fátima Bento ${ }^{V}$
}

\begin{abstract}
RESUMO: Trata-se de estudo quantitativo descritivo transversal, cujo objetivo foi retratar o perfil de saúde-doença dos idosos mais velhos, usuários da atenção básica de saúde. Participaram do estudo 100 idosos de 80 anos ou mais, selecionados mediante critérios de inclusão e exclusão, no período amostral de fevereiro a abril de 2011. Para a coleta dos dados, foi utilizado questionário semiestruturado adaptado e as informações foram analisadas por estatística descritiva. Os resultados apontaram o perfil constituído por idosos longevos sedentários, portadores de doenças crônicas e comorbidades, que referem hábitos saudáveis de vida, avaliam sua saúde como razoável, utilizam diversos medicamentos e possuem como principais atividades de lazer assistir à televisão e realizar serviços domésticos. Os achados permitem constatar a necessidade de estimular atividades que promovam a socialização, já que as trocas de experiências podem proporcionar melhores cuidados individuais, evitar o sedentarismo e intensificar o controle das doenças e dos medicamentos.
\end{abstract}

Palavras-Chave: Idoso de 80 anos ou mais; enfermagem geriátrica; longevidade; centros de saúde.

\begin{abstract}
This quantitative, descriptive, transversal study aimed to profile the health and disease status of older-old primary health care users. Study participants were 100 older adults 80 or more years old, selected on inclusion and exclusion criteria during the sample period from February to April 2011. Data were collected by adapted, semi-structured questionnaire, and analyzed using descriptive statistics. The results revealed a profile consisting of sedentary oldest-old, with chronic diseases and comorbidities, who report healthy living habits, assess their health as fair, use various drugs, and whose main leisure activities are television and household chores. The findings reveal a need to encourage activities that foster socialization, since sharing experience can provide better self-care, prevent sedentary lifestyle, and intensify control of diseases and medicines.

Keywords: Aged, 80 and over; geriatric nursing; longevity; health centers.
\end{abstract}

RESUMEN: Se trata de un estudio cuantitativo descriptivo transversal, cuyo objetivo fue describir el perfil de salud - enfermedad de los ancianos longevos, usuarios de atención primaria de salud. Han participado del estudio 100 ancianos de 80 años o más, seleccionados por medio de criterios de inclusión y exclusión, en el período de muestreo de febrero a abril de 2011. Para recopilar los datos, se adoptó un cuestionario semiestructurado adaptado y se han analizado las informaciones por estadística descriptiva. Los resultados mostraron un perfil constituido de ancianos longevos sedentarios, con enfermedades crónicas y comorbilidades, que señalan tener hábitos de vida saludables, evalúan su salud como regular, utilizan diferentes fármacos y tienen como principales actividades de ocio ver la televisión y realizar tareas domésticas. Los hallazgos ayudan a determinar la necesidad de estimular actividades que promuevan la socialización, ya que el intercambio de experiencias puede ofrecer una mejor atención individual, evitar el sedentarismo e intensificar el control de enfermedades y medicamentos.

Palabras Clave: Anciano de 80 años o más; enfermería geriátrica; longevidad; centros de salud.

\section{INTRODUÇÃO}

A transição demográfica é fenômeno mundial e tem gerado temáticas de discussão, tanto no que se refere à implementação das políticas públicas voltadas para as pessoas idosas como aos serviços e cuidados à saúde na idade avançada.
O aumento da população idosa tem trazido desafios para a sociedade, como a maior carga de doenças, incapacidades e aumento da demanda dos serviços de saúde hospitalares ${ }^{1}$. A cada ano, novos idosos são incorporados à população brasileira e aqueles com 80 anos ou mais já

\footnotetext{
IEnfermeira da Prefeitura Municipal de Colombo. Paraná, Brasil. E-mail: letice freitas@hotmail.com.

IIEnfermeira. Doutora em Filosofia da Enfermagem. Professora Sênior do Programa de Pós-Graduação em Enfermagem da Universidade Federal do Paraná. Líder do Grupo Multiprofissional de Pesquisa sobre Idosos. Curitiba, Paraná, Brasil. E-mail: curitiba.helena@gmail.com.

IIIEnfermeira. Doutora em Enfermagem. Membro do Grupo Multiprofissional de Pesquisa sobre Idosos. Curitiba, Paraná, Brasil. E-mail: tatiane.michel@uol.com.br. IVEnfermeira. Doutoranda do Programa de Pós-Graduação em Enfermagem da Universidade Federal do Paraná. Bolsista Reuni. Membro do Grupo Multiprofissional de Pesquisa sobre Idosos. Curitiba, Paraná, Brasil. E-mail: nathalia.kolb@gmail.com.

vEnfermeira da Prefeitura Municipal de Curitiba. Especialista em Gerenciamento dos Serviços de Enfermagem e Gestão Pública em Saúde e MBA em gestão de
} Recursos Humanos. Curitiba, Paraná, Brasil. E-mail: leandradefatimabento@hotmail.com. 
correspondem a mais de $12 \%$ da população idosa 2 . O Paraná destaca-se como um dos estados do Brasil com maior número de centenários $(933)^{3}$, onde atualmente residem, aproximadamente, 147.193 idosos com 80 anos ou mais, dos quais 27.630 concentram-se na cidade de Curitiba ${ }^{4}$.

A longevidade da população determina importantes repercussões em vários campos, como na área social, da saúde e econômica. A busca da longevidade, de viver mais com saúde e bem-estar constitui um dos principais valores almejados em toda a história da humanidade ${ }^{5}$. No entanto, os problemas de saúde que acometem especialmente os idosos mais velhos ainda são pouco investigados pelos serviços de saúde e pesquisadores; consequentemente, pouco se sabe sobre eles.

Logo, torna-se relevante conhecer as características próprias e condições de saúde-doença dos idosos com idade mais avançada. A obtenção desses dados e sua análise fornecem subsídios, principalmente locais, para o desenvolvimento de novas pesquisas e, ainda, permitem aos profissionais de enfermagem atuar na promoção à saúde e prevenção de doenças e incapacidades, contribuindo para a melhor qualidade de vida dos longevos.

Diante dessas considerações, o objetivo deste estudo foi retratar o perfil de saúde-doença dos idosos mais velhos, usuários da atenção básica de saúde.

\section{REvisão DE Literatura}

Os escassos estudos com essa faixa etária identificam como problemas mais frequentes o declínio cognitivo, presença de comorbidades, dependência funcional e depressão. Tais condições de saúde, além de relacionadas entre si, apresentam-se como importantes preditores de mortalidade ${ }^{6}$. Outros autores apontam o déficit de conhecimento acerca da saúde dos idosos mais velhos, ao ressaltar a carência da produção científica sobre essa faixa etária e a necessidade de os pesquisadores voltarem mais sua atenção para essa temática? . Do mesmo modo, pouco se conhece acerca das características do perfil de saúde-doença dos idosos longevos usuários da atenção básica à saúde. Os institutos de estatísticas nacionais trazem apenas dados da faixa etária com 60 anos ou mais e, frequentemente, não especificam as informações sobre os mais velhos.

O segmento etário dos idosos mais velhos apresenta características morfofisiológicas, psicológicas e socioeconômicas diferenciadas de outros indivíduos, inclusive de idosos mais jovens. Portanto, evidencia-se a heterogeneidade no grupo etário dos idosos, e há diferenças significativas entre aqueles considerados mais jovens (60-79 anos) e os com idade igual ou superior a 80 anos $^{8}$.

\section{Metodologia}

Trata-se de estudo quantitativo descritivo de corte transversal, realizado em uma unidade básica de saúde (UBS) e nos domicílios dos idosos usuários, no
Município de Curitiba, Paraná. A UBS possui área de abrangência de 19 mil habitantes e, aproximadamente, 1600 idosos cadastrados. Diariamente, cerca de 23 idosos recebem algum tipo de atendimento nessa unidade. Em janeiro de 2011, a população de idosos de 80 anos ou mais cadastrados em programas oferecidos pela UBS constituía-se de 109 sujeitos.

Para seleção dos idosos participantes deste estudo, utilizaram-se os seguintes critérios de inclusão: possuir idade igual ou superior a 80 anos; estar cadastrado na UBS selecionada para o estudo; e obter pontuação no Miniexame do Estado Mental (MEEM) acima dos pontos de corte $9: 13$ pontos para analfabetos, 18 pontos com escolaridade baixa e média e 26 pontos para aqueles com escolaridade alta. Foram critérios para exclusão da pesquisa os idosos com dificuldades de comunicação que impossibilitassem responder ao questionário e que não possuíam cuidador familiar para ajudar nas informações.

Dos 109 idosos longevos cadastrados na UBS, seis não residiam mais no bairro, dois não aceitaram participar do estudo e um idoso havia falecido. Portanto, a amostra foi composta por 100 idosos longevos, selecionados de acordo com os critérios de inclusão e exclusão, no período amostral de fevereiro a abril de 2011. Desses, cinco apresentaram dificuldades de comunicação e nove, alteração cognitiva rastreada por meio do MEEM, que os impossibilitou de responder ao questionário. Nesses casos, o cuidador familiar foi convidado a participar do estudo. Foram critérios de inclusão desse cuidador na pesquisa: ser cuidador familiar de idoso com dificuldades de comunicação ou que obteve pontuação no MEEM inferior aos pontos de corte; residir com o idoso longevo; possuir idade igual ou superior a 18 anos.

A coleta de dados ocorreu em fontes primárias e secundárias e foi efetivada em dois momentos: no primeiro, houve consulta aos prontuários eletrônicos e contato telefônico com os idosos longevos potenciais participantes; no segundo, foi feita a aplicação do MEEM e do questionário.

Foram consultados os prontuários dos idosos que possuíam idade igual ou superior a 80 anos e coletados os dados de identificação (nome, idade, endereço e número de telefone). Em seguida, foram realizados contatos telefônicos para verificar a possibilidade e disponibilidade dos idosos participarem como sujeitos da pesquisa. Do total de 100 idosos longevos, 42\% deles responderam ao questionário na UBS e 58\% nos domicílios.

Para a identificação das características do perfil de saúde-doença, foi utilizado um questionário semiestruturado $^{10}$ e adaptado para este estudo, com questões relativas ao estado de saúde, uso de medicamentos, utilização dos serviços de saúde, histórico de quedas, hospitalizações, hábitos de vida e adoção de tecnologias assistivas.

Os dados coletados foram transcritos por digitação, nos programas Excel e Epi Info versão 6.04, anali- 
sados por meio de estatística descritiva e apresentados sob a forma de tabelas.

Foram respeitados os preceitos éticos de participação voluntária e consentida, segundo a Resolução n $^{0}$ 196/96 ${ }^{11}$. O projeto foi aprovado pelo Comitê de Ética do Setor de Ciências da Saúde, conforme parecer com registro $\mathrm{n}^{\mathrm{o}}$ 0107.0.091.091-10.

\section{Resultados e Discussão}

Considerando o perfil de saúde-doença dos 100 idosos longevos, usuários da UBS, participantes deste estudo, 43\% nunca realizaram exercícios físicos; 34\% já os realizaram e hoje não mais; e 23\% ainda os praticam. Desses últimos, 16\% fazem caminhadas, 3\% alongamento, $3 \%$ praticam ginástica e $1 \%$ anda de bicicleta para se exercitar. Ver Tabela 1.

Os resultados mostraram que a maioria dos idosos mais velhos era sedentária (77\%), embora 34\% deles já tivessem feito algum tipo de atividade física no decorrer de sua vida. Dessa forma, considera-se elevada a frequência de sedentarismo na amostra estudada, tendo em vista estudos como o realizado com 129 idosos longevos

TABELA 1: Distribuição percentual das características do perfil de saúde-doença e hábitos de vida dos idosos longevos. Curitiba - PR, 2011. $(N=100)$

\begin{tabular}{|c|c|c|}
\hline Características & Classificação & $\%$ \\
\hline \multirow{3}{*}{$\begin{array}{l}\text { Prática de atividades } \\
\text { físicas }\end{array}$} & Sim, ainda realizo & 23 \\
\hline & Sim, hoje não realizo mais & 34 \\
\hline & Não, nunca realizei & 43 \\
\hline \multirow{3}{*}{$\begin{array}{l}\text { Possui atividades de } \\
\text { lazer }\end{array}$} & Sim & 82 \\
\hline & Não & 18 \\
\hline & Excelente & 3 \\
\hline \multirow{3}{*}{$\begin{array}{l}\text { Considera sua saúde } \\
\text { física }\end{array}$} & Boa & 40 \\
\hline & Razoável & 51 \\
\hline & Não respondeu & 6 \\
\hline \multirow{2}{*}{$\begin{array}{l}\text { Participa de algum } \\
\text { grupo }\end{array}$} & Sim & 13 \\
\hline & Não & 87 \\
\hline \multirow{2}{*}{$\begin{array}{l}\text { Possui alguma } \\
\text { doença }\end{array}$} & Sim & 91 \\
\hline & Não & 9 \\
\hline \multirow{3}{*}{$\begin{array}{l}\text { Toma algum } \\
\text { medicamento }\end{array}$} & Sim & 95 \\
\hline & Não & 5 \\
\hline & Público & 68 \\
\hline \multirow{3}{*}{$\begin{array}{l}\text { Serviço de saúde } \\
\text { utilizado }\end{array}$} & Público - Convênio & 18 \\
\hline & Público - Particular & 10 \\
\hline & $\begin{array}{l}\text { Público - Convênio - } \\
\text { Particular }\end{array}$ & 4 \\
\hline \multirow{3}{*}{$\begin{array}{l}\text { Foi hospitalizado nos } \\
\text { últimos } 12 \text { meses }\end{array}$} & Sim & 25 \\
\hline & Não & 75 \\
\hline & Ex - Tabagista & 9 \\
\hline \multirow[t]{2}{*}{ Tabagista } & Sim & 6 \\
\hline & Não & 85 \\
\hline \multirow{2}{*}{ Etilista } & Sim & 5 \\
\hline & Não & 95 \\
\hline
\end{tabular}

moradores de São Geraldo, no Estado de Minas Gerais $(\mathrm{MG})^{12}$, que apontou 31\% dos longevos sedentários.

Existe tendência de redução do nível de atividade física com o aumento da idade, principalmente entre as mulheres longevas ${ }^{12}$. O sedentarismo no idoso longevo pode ser explicado, em parte, pelo próprio processo de envelhecimento fisiológico que, com o passar dos anos, resulta na diminuição da massa muscular, perda de massa óssea, atrofia muscular e dificulta ou reduz a prática de atividade física.

A atividade física pode contribuir para as pessoas idosas ficarem independentes o máximo possível, por período de tempo mais longo, e reduzir o risco de quedas nesta população. Por isso, a prática de atividades deve ser priorizada, também, nas idades mais avançadas, levando em consideração seus possíveis benefícios ${ }^{13,14}$.

As principais atividades de lazer exercidas pelos idosos longevos foram assistir à televisão (29\%), seguida dos serviços domésticos (19\%) e trabalhos manuais (15\%). Entre as atividades menos realizadas destaca-se viajar, citada por apenas 3\% participantes.

Uma vida ativa melhora a saúde mental e, frequentemente, promove contatos sociais ${ }^{13}$. Entretanto, observou-se, na presente investigação, que as atividades de lazer são realizadas pela maioria dos longevos no próprio domicílio e, desse modo, favorecem ainda mais o sedentarismo e o isolamento social. Achado semelhante foi encontrado em estudo com $271 \mathrm{mu}$ lheres octogenárias residentes em Uberaba (MG), no qual 79,3\% referiram assistir à televisão e 59,5\% ouvir rádio, sendo que $70,4 \%$ delas estão satisfeitas com as atividades de lazer que realizam ${ }^{15}$.

Destaca-se, ainda, que apenas 12\% dos idosos longevos deste estudo participavam de grupos de convivência como os da terceira idade e de voluntários e $1 \%$ de grupo terapêutico para hipertensão. A baixa frequência de participação de idosos em grupos foi encontrada igualmente em outros estudos, como na pesquisa desenvolvida com 197 idosos, de ambos os sexos, em Belo Horizonte (MG), na qual os longevos representaram a faixa etária de menor frequência no grupo $^{16}$. Entre as justificativas que limitam a participação deles estão maior grau de dependência e comorbidades $^{16}$, dificuldades de acesso e falta de informação das atividades voltadas a eles ${ }^{17}$.

Para um envelhecimento ativo, é preciso apoiar e permitir a participação ativa de pessoas idosas nas atividades de desenvolvimento econômico, trabalho formal e informal e atividades voluntárias, de acordo com suas necessidades individuais, preferências e capacidades ${ }^{13}$. Além disso, o ano 2012 foi marcado pelo Ano Europeu do Envelhecimento Ativo e Solidariedade entre Gerações, que teve como objetivo primordial "sensibilizar, difundir as boas práticas e incentivar os responsáveis políticos e as partes interessadas a facilitar o envelhecimento ativo"18:9. Dessa maneira, é esperado que os 
governos dos países que compõem a União Europeia desenvolvam ações para incluir as pessoas idosas na sociedade de forma mais ativa. Tais iniciativas podem servir de exemplo para o Brasil.

Para a maioria (51\%) dos idosos longevos deste estudo, a saúde foi avaliada como razoável, 40\% consideram boa e apenas 3\% a avaliaram como excelente, conforme a Tabela 1. A autoavaliação de saúde tem sido utilizada como indicador de bem-estar nas pesquisas com pessoas idosas e considerada preditora de morbidade, incapacidade e inatividade. Esses fatores podem contribuir para a pior autoavaliação da saúde na população com 80 anos ou mais; entretanto, poucas pesquisas têm enfocado este segmento etário.

Em inquérito populacional no município de Campinas, dos 1.432 idosos longevos pesquisados, $64,5 \%$ avaliaram a sua saúde como boa, 24,6\% como excelente ou muito boa e apenas 10\% a consideraram ruim $^{19}$. Tais achados contrastam com os resultados da presente pesquisa.

O quantitativo de idosos mais velhos que avaliaram sua saúde como boa foi expressivo neste estudo; no entanto, todos referiram uma ou mais doenças crônicas. Dos 100 participantes, apenas 9\% negaram qualquer tipo de doença, enquanto 91\% afirmaram possuir uma ou mais, e relataram até nove comorbidades. A distribuição das doenças referidas pelos longevos mostrou destaque para hipertensão arterial (67\%), problemas osteomusculares (39\%), seguido de diabetes mellitus (27\%) e doenças cardiovasculares (20\%).

A autopercepção da saúde torna-se prejudicada nos casos de doenças que, frequentemente, não se manifestam com intensidade e que costumam ser mais silenciosas, como a hipertensão. Ainda, as doenças referidas são de caráter crônico, o que pode tornar o idoso acostumado com os sinais e sintomas, uma vez que a medicação minimiza a sintomatologia.

Os idosos na faixa etária de 80 anos ou mais têm como característica clínica a maior frequência de doenças crônicas. Os resultados da presente investigação convergem para os achados de estudos nacionais e, entre eles, destaca-se a pesquisa realizada com 2.136 idosos residentes em São Paulo que, de forma semelhante, revelou a hipertensão $(53,3 \%)$ e as doenças reumáticas $(38,3 \%)$ como as de maiores prevalências nesta população ${ }^{20}$.

A maioria dos idosos longevos (95\%) relatou fazer uso de uma ou mais medicações, segundo a Tabela 1. Eles utilizam, em média, 2,6 medicamentos (mínimo=1 e máximo=11), com destaque para os anti-hipertensivos (83\%), diuréticos (26\%), antiagregantes plaquetários (18\%), antilipêmicos (18\%) e analgésicos (17\%).

A polifarmácia tem sido associada à idade avançada em diversos estudos ${ }^{12,21}$. Essas pesquisas apontam que os idosos apresentam aumento da frequência das doenças crônico-degenerativas, cujo controle e prevenção de sequelas, muitas vezes, demandam o uso constante de medicamentos. Um desses estudos ${ }^{21}$, cujo objetivo foi caracterizar o uso de medicamentos por 667 idosos residentes em Belo Horizonte (MG), apresentou associação estatística significativa entre uso de medicamentos e idade maior ou igual a 80 anos.

A utilização concomitante de vários medicamentos pelos longevos implica a atenção dos profissionais quanto ao uso inadequado, o qual pode oferecer sérios riscos à saúde desses idosos. A iatrogenia induzida por medicamentos é comum em idades avançadas e pode ocorrer devido a interações medicamentosas, dosagens inadequadas, reações imprevisíveis e quedas associadas ao uso de medicamentos como os psicotrópicos ${ }^{13}$. A incidência de reações indesejadas decorrentes da administração de medicamentos aumenta proporcionalmente à idade 2 . Os idosos longevos, frequentemente, apresentam diversos fatores que podem predispor aos efeitos indesejados das terapias farmacológicas. Desse modo, ressalta-se a utilização criteriosa e controlada de fármacos nos longevos e as orientações dos profissionais tanto a eles quanto a seus familiares.

Quanto à utilização dos serviços de saúde, neste estudo, observou-se que 68\% dos idosos longevos utilizam somente o serviço público de saúde e $25 \%$ foram hospitalizados nos últimos 12 meses, de acordo com a Tabela1. Esses dados são semelhantes aos encontrados entre 271 mulheres com 80 anos ou mais residentes na zona urbana de Uberaba (MG), no qual 50\% referiram utilizar, principalmente, os serviços públicos de saúde e 31,1\% estiveram internadas nos últimos 12 meses ${ }^{15}$. Dessa forma, sendo o serviço público de saúde o mais utilizado pelos idosos - e como o Brasil será o sexto país em número de idosos até $2025^{13}$-, observa-se a necessidade de adequada preparação dos centros de saúde, bem como dos profissionais, para enfrentamento e conhecimento das demandas causadas pelo envelhecimento da população.

Concernente aos hábitos de vida, 85\% dos participantes relataram não fumar; $9 \%$ são ex-tabagistas há mais de 20 anos e $6 \%$ ainda fumam. Desses, 2\% fumam há mais de 30 anos e 4\% são tabagistas há menos de 30 anos. O etilismo foi reportado por 5\% dos longevos.

Os participantes deste estudo alegaram possuir hábitos de vida saudáveis. Outro estudo, realizado com sujeitos dessa faixa etária confirma essas alegações ${ }^{20}$. No entanto, não se sabe qual é o entendimento dos idosos mais velhos sobre hábitos saudáveis e entende-se que o real sentido atribuído por eles envolve questões culturais. Acredita-se que as pesquisas etnográficas poderiam contribuir com mais propriedade para o estudo dessa questão.

Quanto às tecnologias assistivas para locomoção, a maior parte (73\%) dos longevos não as utiliza, 21\% usam bengalas, $5 \%$ transportam-se em cadeira de rodas e $1 \%$ encontrava-se acamado. Dos 100 idosos pesquisados, 31\% caíram nos últimos 12 meses. Ainda 69\% deles referiram o uso de óculos, sendo $15 \%$ apenas para ler, e 85\% não utilizam aparelho para surdez. Ver Tabela 2. 
TABELA 2: Distribuição percentual do uso de tecnologias assistivas e condição bucal dos idosos longevos. Curitiba - PR, 2011. ( $N=100)$

\begin{tabular}{llc}
\hline Características & \multicolumn{1}{c}{ Classificação } & \% \\
\hline \multirow{4}{*}{$\begin{array}{c}\text { Tecnologias assistivas } \\
\text { para deambulação }\end{array}$} & Mengala & 21 \\
& Cadeta & 0 \\
& Acamado de rodas & 5 \\
& Não utilizam & 1 \\
\multirow{3}{*}{ Oculos } & Sim & 73 \\
& Só para ler & 54 \\
Aparelho de surdez & Não & 15 \\
& Sim & 31 \\
& Não & 15 \\
Dentes & Têm todos & 85 \\
& Têm poucos dentes & 4 \\
& Não têm dentes & 5 \\
& Usa dentadura & 83 \\
\hline
\end{tabular}

Nesta pesquisa, observou-se que a maioria dos idosos mais velhos não utiliza nenhum dispositivo para auxílio na locomoção, tampouco aparelhos para surdez; no entanto, foi detectada, em vários deles, a necessidade desse recurso. Estudo desenvolvido com 137 residentes, no Rio Grande do Sul, para avaliar as condições de vida, saúde e apoio dos idosos mais velhos, revelou que $27,7 \%$ utilizam bengalas e $27,7 \%$ se encostam em móveis e paredes como meio de apoio para locomoção ${ }^{22}$.

Entende-se que o uso de dispositivos auxiliares da marcha pode fazer a diferença na manutenção da independência e autonomia entre os idosos. A utilização de tecnologias assistivas pode melhorar a capacidade dos longevos para realizar as atividades diárias de maneira o mais independente possível. A compensação de deficiências é amplamente recomendada para o envelhecimento ativo ${ }^{13}$, podendo envolver vários acessórios, adaptações e tecnologias, como bengala, andador, óculos, aparelho de surdez, corrimãos, entre outros.

Estudos utilizados em uma revisão da literatura demonstraram a baixa utilização de aparelhos para surdez pelos idosos de 80 anos ou mais. Ressaltam que a perda auditiva, associada ao envelhecimento, é um fenômeno com alta prevalência na população idosa, podendo ocasionar uma série de dificuldades na comunicação oral, bem como, muito frequentemente, na interação familiar e social ${ }^{23}$.

O edentulismo é frequente nas pessoas com idade mais avançada e, neste estudo, encontrou-se que 83\% dos longevos afirmaram utilizar prótese dentária e apenas 4\% possuir todos os dentes (Tabela 2). Esses resultados são semelhantes ao verificado em uma pesquisa de revisão bibliográfica ${ }^{24}$, a qual apresentou que a maior parte dos idosos mais velhos tem menos da metade dos dentes; muitos deles utilizam dentaduras e nenhum foi observado com todos os dentes preservados.

Estudos demonstram que uma saúde oral precária está associada à desnutrição e, portanto, ao maior risco de desenvolver várias doenças não transmissíveis e a pior qualidade de vida ${ }^{13,25}$. Em uma dessas pesquisas, os autores ${ }^{25}$ constataram que quanto maior a idade, menor o uso de serviços odontológicos.

Quanto às características da alimentação referida pelos longevos, a maioria deles alegou possuir uma alimentação saudável com a ingestão de frutas (68\%), verduras (77\%), leite e derivados (74\%), carne branca e vermelha (62\%), e 1\% recebe dieta especial via gastrostomia, conforme a Tabela 3.

TABELA 3: Distribuição percentual dos tipos de alimentos consumidos por idosos longevos. Curitiba - PR, 2011. ( $N=100)$

\begin{tabular}{lccc}
\hline \multicolumn{1}{c}{ Alimentação(*) $^{*}$} & Sempre & $\begin{array}{c}\text { Às } \\
\text { vezes }\end{array}$ & Pouco \\
\hline Ingestão de frutas & 68 & 10 & 21 \\
Ingestão de verduras & 77 & 6 & 16 \\
Ingestão de leite e derivados & 74 & 5 & 20 \\
Ingestão de carne/frango/peixe & 62 & 11 & 26 \\
\hline
\end{tabular}

${ }^{(*)} \mathrm{Um}$ idoso longevo recebe dieta especial via gastrostomia.

Um dos fatores relacionados ao envelhecimento sadio é a boa nutrição durante toda a vida. $\mathrm{O}$ estado nutricional adequado aumenta a possibilidade das pessoas se aproximarem do seu ciclo máximo de vida $^{13,26}$. Em estudo qualitativo realizado com 14 idosos longevos na Austrália, os participantes consideraram essencial uma dieta balanceada para um bom estado de saúde e referiram que uma boa alimentação é uma dieta rica em frutas e verduras e com redução de carne $^{27}$. Observou-se no presente estudo que a maior parte dos idosos longevos referiu se alimentar com frutas, verduras, leites e carnes. Entretanto, como se trata de autorrelato, as questões referentes à alimentação dos idosos longevos poderiam ser mais exploradas mediante estudos etnográficos.

\section{Conclusão}

perfil dos participantes deste estudo mostra longevos que alegam bons hábitos de vida, com baixa frequência de etilismo e tabagismo e alimentação saudável. Em contrapartida, são sedentários e carentes de atividades que promovam a socialização e um envelhecimento ativo, visto que não participam de grupos de convivência e apresentam como principais meios de lazer a televisão e os serviços domésticos.

Para a enfermagem, a socialização do longevo por meio de grupos de convivência tem como intuito desenvolver o sentimento coletivo da solidariedade, alicerçado nas trocas de experiências de cuidado. O espírito de cooperação que se desenvolve pela integração mais intensa entre eles intensifica o entusiasmo pela vida e desejos de se cuidar cada vez mais e melhor. 
Infere-se que somente os hábitos saudáveis alegados pelos longevos não são suficientes para evitar a incidência de doenças e seus agravos, visto que possuem uma ou mais doenças crônicas, com destaque para a hipertensão arterial sistêmica e doenças osteomusculares. Ainda, a presença da polifarmácia é um dado frequente nos resultados de pesquisas nacionais com esta faixa etária e um alerta para a atenção dos profissionais quanto ao uso benéfico dos medicamentos.

No presente estudo, alguns longevos afirmaram não possuir doenças; entretanto, utilizam medicamentos. Isso mostra a necessidade do olhar atento do profissional de enfermagem para as várias dimensões das orientações de cuidado. Centrar a ação profissional nos problemas clínicos é fundamental, bem como considerar os atos provenientes de questões culturais que se desenvolvem em razão do pertencimento familiar do longevo, como o hábito da automedicação.

Um dos modos para reduzir as deficiências entre os longevos é facilitar o acesso de todos aos aparelhos e tecnologias apropriadas. Muitos dos idosos não os utilizavam por apresentar dificuldades no processo de aquisição desses recursos, entre as quais a morosidade e o alto custo. Do mesmo modo, não se pode aceitar o edentulismo como um fenômeno normal e natural do envelhecimento, mas como reflexo da falta de políticas de saúde preventivas, destinadas à população adulta para a manutenção dos dentes até a idade avançada.

Este estudo possui limitações em virtude da escassa produção científica acerca do segmento etário dos longevos e da reduzida amostra, o que dificultou a inferência sobre certas variáveis. Outros estudos quantitativos com amostras significativas poderão aprofundar os achados e, por meio de pesquisas que utilizam abordagens qualitativas, elucidar questões subjetivas envolvidas na saúde e doença dos idosos longevos.

\section{REFERÊNCIAS}

1.Veras R. Envelhecimento populacional contemporâneo: demandas, desafios e inovações. Rev Saude Publica. 2009; 43:548-54.

2.Ministério da Saúde $(\mathrm{Br})$. Secretaria de Atenção à Saúde, Departamento de Atenção Básica. Envelhecimento e saúde da pessoa idosa. Brasília (DF): Editora MS; 2010. 3.Instituto Brasileiro de Geografia e Estatística . Censo Demográfico 2010. Características da População e dos Domicílios: Resultados do Universo [site de internet]. Brasil(DF): IBGE; 2010. [citado em 08 mai 2015]. Disponível em: http:/www.ibge.gov.br/estadosat/temas.php $?$ sigla $=$ pr\&tema $=$ resultuniverso_censo2010.

4.Ministério da Saúde (Br). Tecnologia da informação a serviço do SUS - DATASUS. Projeções intercensitárias [site de internet]. Brasilia(DF): DATASUS; 2012. [citado em 08 mai 2015]. Disponível em: http://tabnet. datasus.gov.br/cgi/tabcgi.exe?ibge/cnv/poppr.def 5.Francisco CM. Estudo dos fatores preditores de en- velhecimento sem incapacidade funcional entre idosos em velhice avançada no município de São Paulo [dissertação de mestrado]. São Paulo: Universidade de São Paulo; 2006.

6.Rosset I, Pedrazzi EC, Roriz-Cruz M, Morais EP, Rodrigues RAP. Tendências dos estudos com idosos mais velhos na comunidade: uma revisão sistemática (inter) nacional. Rev esc enferm USP. 2011; 45:264-71

7.Menezes TM, Lopes RLM. Produção do conhecimento sobre idoso longevo: 1998-2008. Rev enferm UERJ. 2009; 17:569-74.

8.Marafon LP, Cruz IBM, Schwanke CHA, Moriguchi EHM. Preditores cardiovasculares da mortalidade em idosos longevos. Cad Saúde Pública. 2003; 19:799-807. 9.Bertolucci PH, Brucki SM, Campacci SR, Juliano Y. The Mini-Mental State Examination in a general population: impact of educational status. Arq Neuro-Psiquiatr. 1994; 52:1-7.

10.Instituto Brasileiro de Geografia e Estatística (Br). Censo demográfico 2000. Questionário da Amostra CD 1.02. [site de internet]. Brasil(DF): IBGE; 2000. [citado em 10 nov 2010]. Disponível em: http://www.ibge.gov. br/home/estatistica/populacao/censo2000/migracao/ censo2000_migracao.pdf.

11.Conselho Nacional de Saúde $(\mathrm{Br})$. Resolução $\mathrm{n}^{\mathrm{O}}$ 196, de 10 de outubro de 1996. Aprova as Diretrizes e Normas Regulamentadoras de Pesquisas envolvendo Seres Humanos. [site de internet]. Brasília: Ministério da Saúde, 1996. [citado em 10 nov 2014]. Disponível em: http://dtr2004.saude.gov.br/susdeaz/legislacao/arquivo/ Resolucao_196_de_10_10_1996.pdf

12. Nogueira SL, Ribeiro RCL, Rosado LEFPL, Franceschini SCC, Ribeiro AQ, Pereira ET. Fatores determinantes da capacidade funcional em idosos longevos. Rev Bras Fisioter. 2010; 14:322-9.

13.Organização Mundial da Saúde. Envelhecimento ativo: uma política de saúde. Brasília (DF): Organização Pan Americana de Saúde; 2005.

14.Honório GJS, Martins HEL, Basso JF, Alvarez AM, Meirelles BS, Santos SMA. Estratégias de promoção da saúde dos idosos no Brasil: um estudo bibliométrico. Rev enferm UERJ. 2013;21:121-6.

15.Soares MBO, Tavares DMS, Dias FA, Diniz MA, Machado ARM. Características sociodemográficas, econômicas e de saúde de idosas octogenárias. Cienc Cuid Saúde. 2009; 8:452-9.

16. Borges PLC, Bretas RP, Azevedo SF, Barbosa JMM. Perfil dos idosos frequentadores de grupos de convivência em Belo Horizonte, Minas Gerais, Brasil. Cad Saúde Pública. 2008; 24:2798-808.

17.Peregrino AAF, Schutz V, Marta CB, Pereira ACA, Silva GP, Nogueira LC. Buscando a inserção dos idosos nas ações de promoção social e de saúde. Rev enferm UERJ. 2012; 20:513-8.

18.European Union. Active ageing and solidarity between generations : a statistical portrait of the European Union 2012. Bélgica: Eurostat; 2011.

19.Borim FSA, Barros MBA, Neri AL. Autoavaliação da saúde em idosos: pesquisa de base populacional no 
município de Campinas, São Paulo, Brasil. Cad Saúde Pública. 2012; 28:769-80.

20.Ferreira JVC. Os muito idosos no município de São Paulo [dissertação de mestrado]. São Paulo: Universidade de São Paulo; 2006.

21.Ribeiro AQ, Rozenfeld S, Klein CH, Cesar CC, Acurcio FA. Inquérito sobre uso de medicamentos por idosos aposentados, Belo Horizonte, MG. Rev Saude Publica. 2008; 42:24-32.

22.Morais EP. Envelhecimento no meio rural: condições de vida, saúde e apoio dos idosos mais velhos de Encruzilhada do Sul - RS [tese de doutorado]. Ribeirão Preto (SP): Universidade de São Paulo; 2007.

23. Veras RP, Mattos LC. Audiologia do envelhecimento: revisão da literatura e perspectivas atuais. Rev Bras
Otorrinolaringol. 2007; 73:128-34.

24.Ferrari MAC. Idosos muito idosos: reflexões e tendências. O Mundo da Saúde. 2002; 26:467-71.

25.Matos DL, Giatti L, Lima-Costa MF. Fatores sociodemográficos associados ao uso de serviços odontológicos entre idosos brasileiros: um estudo baseado na Pesquisa Nacional por Amostra de Domicílios. Cad Saúde Pública. 2004; 20:1290-7.

26.Campos MAG, Predoso ERP, Lamounier JA, Calosimo EA, Abrantes MM. Estado nutricional e fatores associados em idosos. Rev Assoc Med Bras. 2006; 52:214-21. 27. Orb A. Aspectos de salud em adultos mayores de 80 anos de edad que viven independientemente en la comunidad: uma perspectiva australiana. Rev Latino-Am Enfermagem. 2004; 12:589-96. 